\title{
Efficacy of the levonorgestrel-releasing intrauterine device is associated with different subtypes of adenomyosis: a retrospective study
}

\author{
Shuyi Chen, Jianzhang Wang, Wenting Sun, Libo Zhu, Jiayi He, Xinmei Zhang \\ The Department of Gynecology, Women's Hospital, Zhejiang University School of Medicine, Hangzhou, China \\ Contributions: (I) Conception and design: S Chen, X Zhang; (II) Administrative support: X Zhang; (III) Provision of study materials or patients: All \\ authors; (IV) Collection and assembly of data: S Chen, J Wang, W Sun, J He; (V) Data analysis and interpretation: All authors; (VI) Manuscript \\ writing: All authors; (VII) Final approval of manuscript: All authors. \\ Correspondence to: Xinmei Zhang, MD, PhD. The Department of Gynecology, Women's Hospital, Zhejiang University School of Medicine, No. 1 \\ Xueshi Road, Hangzhou 310006, China. Email: zhangxinm@zju.edu.cn.
}

Background: Although the levonorgestrel-releasing intrauterine device (LNG-IUD) has been widely applied in the treatment of adenomyosis, not all the patients are satisfied with its efficacy. The present retrospective study aimed to investigate the efficacy of LNG-IUD on different subtypes of adenomyosis.

Methods: The study comprised a cohort of 207 patients who received the LNG-IUD at the Women's Hospital, Zhejiang University School of Medicine, China, from June 2013 to June 2016. Different subtypes of adenomyosis were classified by magnetic resonance imaging (MRI) and patients were subcategorized into three groups (subtype I: intrinsic, $n=70$; subtype II: extrinsic, $n=73$; subtype IV: indeterminate, $n=64$ ). Multiple variables were compared among the different groups.

Results: Patient demographics, clinical features and the treatment effects of the LNG-IUD were compared between the three subtype groups. The numeric rating scale (NRS) and pictorial blood loss assessment chart (PBAC) score markedly decreased after insertion of the LNG-IUD compared with baseline in all patients in the three subtype groups ( $\mathrm{P}<0.001$ for all groups). Compared to the other two subtypes, the efficacy rate was lower and the spontaneous expulsion rate was higher in subtype IV adenomyosis patients than that in other two groups $(\mathrm{P}<0.05)$. The independent factor associated with the spontaneous expulsion of the system was suggested to be uterine size before IUD placement and bleeding amount after LNG-IUD treatment in the subtype I [P=0.029, hazards ratio (HR): 3.37, 95\% confidence interval $(\mathrm{CI}): 1.09-6.88]$ and IV $(\mathrm{P}=0.045$, HR: 1.02, 95\% CI: 1.01-1.21) adenomyosis patients respectively.

Conclusions: The LNG-IUD is proved to be an effective approach to treat subtype I and II adenomyosis. However, further study is warranted to explore a more suitable protocol to treat this subtype IV adenomyosis due to the high incidence of treatment failure and expulsion.

Keywords: Adenomyosis; levonorgestrel-releasing intrauterine device (LNG-IUD); magnetic resonance imaging classification (MRI classification); treatment failure; expulsion

Submitted Apr 22, 2020. Accepted for publication Sep 13, 2020.

doi: $10.21037 / \mathrm{atm}-20-3420$

View this article at: http://dx.doi.org/10.21037/atm-20-3420

\section{Introduction}

Adenomyosis is a common estrogen-dependent, a benign gynecological disease characterized by endometrial glands and stroma invading, implanting, and proliferating in the myometrium to form diffuse or localized lesions. Its primary clinical symptoms are dysmenorrhea, menorrhagia, and infertility in women of reproductive age (1). Nevertheless, the etiology and pathogenesis of this disorder remain 
unclear. The reported prevalence of adenomyosis is variable because of different diagnostic criteria and bias. The noninvasive diagnosis of adenomyosis mainly depends on transvaginal ultrasonography (TVS) and magnetic resonance imaging (MRI). Ultrasound examination is relatively cheap and enables quick diagnosis; however, diagnostic criteria of multiple features have not been standardized and validated (2). MRI has been shown to have diagnostic accuracy owing to objective image findings. Several additional features can be obtained to distinguish four subtypes by MRI (3).

Several recent studies have attempted to standardize adenomyosis imaging results in a clinically useful classification system. Kishi et al. proposed a classification scheme based on the location relationship between adenomyosis lesions and the uterine structural components (4). The classification of adenomyosis depending on MRI is considered controversial (5); however, we found that various subtypes of adenomyosis contributed to different therapeutic responses and satisfaction in clinical practice.

Depending on disease development and patients' desire to conceive, many conservative surgical choices, including lesion resection, endometrial ablation, uterine artery embolization (UAE), and high-intensity focused ultrasound (HIFU), are offered to relieve severe symptoms. However, women with adenomyosis, who have residual lesions, generally require long-term medical management after conservation surgery, and medication is the mainstream treatment. Gonadotropin-releasing hormone agonist (GnRHa) cannot be used for long periods due to its high price and multiple side-effects, including reduced estrogen levels (6). The use of drugs, such as dienogest and oral contraceptives, which are effective among women of other cultures, still require investigation among Chinese women who are mostly unwilling to accept them for long-term use (7).

The levonorgestrel-releasing intrauterine device (LNGIUD; Mirena, Bayer Healthcare) is widely used for its therapeutic effects, including the release of synthetic progesterone at a rate of $20 \mathrm{ug} / \mathrm{day}$. Many published reports have verified its long-term effects in the treatment of uterine adenomyosis over 3 years. In their study, Sheng et al. reported that the overall effective rate of 3 years was as high as $72.5 \%$ (8); however, some patients still complained of unchanged or worsening symptoms, including severe symptoms. The side-effects of LNGIUD include spontaneous expulsion and prolonged irregular vaginal bleeding, which can cause patient dissatisfaction. In the present study, we analyzed the specific characteristics of each subtype of adenomyosis based on MRI. We evaluated the relationship between subtypes and LNG-IUD expulsion, efficacy, and sideeffects in these patients to improve treatment effects for the long-term management of women with different subtype adenomyosis. We present the following article in accordance with the STROBE reporting checklist (available at: http://dx.doi.org/10.21037/atm-20-3420).

\section{Methods}

\section{Patients}

The present retrospective study was conducted in the Department of Gynecology at Women's Hospital, Zhejiang University School of Medicine in China from June 2013 to June 2016. The Ethics Committee of Women's Hospital, Zhejiang University School (No. IRB-20190089-012) approved the study in accordance with the ethical standards in the Declaration of Helsinki (as revised in 2013) and waived the informed consent.

\section{Categorization of adenomyosis by MRI}

The classification was based on the geographic relationship between adenomyosis lesions and other physiological components of the uterine by experienced radiologists in our hospital. The diagnosis and categorization of adenomyosis by MRI was established when the agreement of the common diagnosis and subtype by the three radiologists was reached. We defined subtype I (intrinsic) as affecting the endometrium and the junctional zone of the uterus; subtype II (extrinsic) as having a disrupted outer shell of the uterus, but unaffected inner components; subtype III (intramural) as residing locally in the myometrium; and subtype IV (indeterminate) as the remaining lesions (Figure 1).

\section{Inclusion and exclusion criteria}

The inclusion criteria were as follows: (I) patients complaining of dysmenorrhea and/or menorrhagia. We defined dysmenorrhea as a numeric rating scale (NRS) score from 1 to 10 , and menorrhagia as a pictorial blood loss assessment chart (PBAC) score $>100$; (II) diagnosis by both ultrasound and MRI criteria (9); (III) premenopausal 

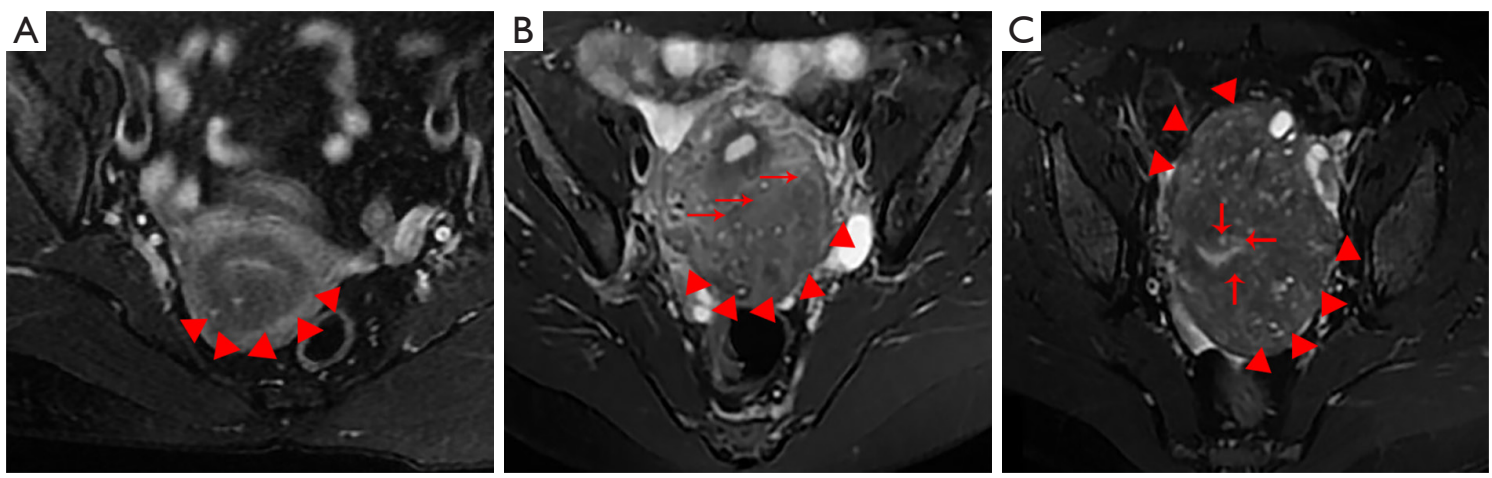

Figure 1 Magnetic resonance imaging (MRI) characteristics of different subtypes of non-focal adenomyosis. T2-weighted MRI of intrinsic (A), extrinsic (B), and indeterminate (C) adenomyosis. (A) Adenomyosis lesions reside within the junctional zone (JZ) (triangle); (B) adenomyosis foci are seen in the outer myometrium, and the serosal layer is destroyed (triangle and arrow); (C) anterior and posterior hemispheres of the uterus are damaged by adenomyosis, including the JZ (triangle and arrow).

women with a menstrual cycle of 21-35 days who wanted to preserve the uterus; (IV) uterine volume $<12$ gestational weeks by ultrasound; (V) LNG-IUD treatment after MRI examination; and (VI) presence or absence of endometriosis, as diagnosed by MRI and laparoscopy. Endometriosis patients had lesions removal surgery before or during the LNG-IUD insertion. The exclusion criteria were: (I) presence of leiomyoma with a maximum diameter $>5 \mathrm{~cm}$; (II) previous treatment of adenomyosis foci removal surgery or high-intensity focused ultrasound; (III) subtype III adenomyosis: intramural; (IV) current or a history of cancer (e.g., breast cancer); (V) hormone treatment, except GnRHa or surgical intervention while the LNG-IUD was in place; (VI) pelvic pain with unknown cause; and (VII) incomplete information.

\section{Evaluation of clinical characteristics}

We collected the demographic records and clinical characteristics of the enrolled patients through the hospital record system and performed a telephone interview to obtain the extra data 3 months after the LNG-IUD insertion. The NRS was used to assess pain intensity on an 11-piont verbal pain rating scale (range, $0-10$ ). Scores were graded as none [0], mild [1-3], moderate [4-6], and severe [7-10] (10). Menstrual blood loss was estimated by PBAC (11). The quantification of menstrual blood loss, including any blood clots, was calculated. A chart score $>100$ was judged as menorrhagia, which was equal to a blood loss amount $>80 \mathrm{~mL}$. Patients were interviewed at 1-, 2-, and 3-year intervals after insertion of the LNG-
IUD to obtain variable data. Uterine volume was calculated by three-dimensional ultrasound examination before the device insertion according to the following formula: volume $=\mathrm{A} \times \mathrm{B} \times \mathrm{C} \times 0.52$, where $\mathrm{A}, \mathrm{B}$ and $\mathrm{C}$ represented diameters of the sphere in each of the three planes in the uterus, respectively (12).

The normal frequency was defined as bleeding or spotting three-to-five times within the relevant 90-day period; amenorrhea was defined as no bleeding during 90-day treatment; Oligomenorrhea was defined as vaginal bleeding less than three times during the 90 days; prolonged bleeding was defined as vaginal bleeding lasting $\geq 14$ days during the 90 days; and frequent bleeding was defined as bleeding six or more times during the 90 days (13).

In the present study, the therapeutic efficacy of the LNG-IUD was evaluated as follows: (I) complete remission: dysmenorrhea and menorrhagia disappeared completely after insertion; (II) significant remission: pain score (NRS score) decreased by more than three grades, and/or menstrual loss amount (PBAC score) reduced to half, but symptom did not completely disappear after treatment; (III) partial remission: pain score reduced by less than two grades and/or menstrual loss amount decreased to more than half of what it used to be; (IV) no remission: pain score or menstrual loss amount was unchanged after the LNGIUD; and (V) recurrence: symptom remission was achieved, but dysmenorrhea or menorrhagia recurred and was progressively aggravated after treatment (14). Consequently, we defined complete and significant remission as clinically effective treatment, and partial and no remission was defined as ineffective treatment. 
The device was inserted into the uterine cavity during GnRHa treatment in patients with amenorrhea, those who had undergone laparoscopic endometriotic lesion removal, or patients on days 5-7 of their menstrual cycle. Three months after device insertion was considered as a stable time. Removal was defined as intentionally taking out the IUD due to poor treatment effects or adverse effects. The expulsion was defined as an unintentional loss of device from the uterine cavity. We confirmed IUD expulsion as either the patient reporting expulsion from the vagina or ultrasound, showing no device in the uterine cavity.

\section{Statistical analysis}

Statistical analysis was performed using SPSS software (version 22.0). Continuous results were all expressed as mean \pm standard deviation, and categorical variables were expressed as number or percentage. Kruskal-Wallis test compared data, followed by Dunn post-hoc test and analysis of variance among the three subtype groups with non-normal and normal distributions. $\chi^{2}$-test or Fisher's exact test was used for categorical variables. Device expulsion was analyzed by menstrual blood loss before treatment, menstrual blood loss after treatment, uterine volume, GnRHa therapy, co-existing leiomyoma, and insertion time. Univariate and multivariate Cox regression analyses were used to identify the relationship between covariates and expulsion of the LNG-IUD after insertion. The variables that were calculated with $\mathrm{P}<0.1$ in the univariate analysis were evaluated in the multivariate Cox regression analysis. Sensitivity analyses were performed by stratified subgroup repeated Cox regression and bootstrap resampling procedure. The results were reported as $\mathrm{P}$ values, hazard ratios (HRs), and 95\% confidence intervals (CIs). All statistical tests were two sides, with $\mathrm{P}<0.05$ as the cut-off level for significance.

\section{Results}

\section{Comparison of clinical characteristics of the three participant subtypes}

As shown in Figure 2, a total of 281 adenomyosis patients who received the LNG-IUD for therapy were included in the present study. These patients underwent an MRI examination before LNG-IUD treatment and were followed by telephone interview. Of the 281 patients, we excluded 32 with subtype III disease and 42 who did not meet the inclusion criteria. Consequently, 207 patients remained and were classified into three groups: subtype I, II, or IV in our study. A total of 189 (91.30\%) patients had severe dysmenorrhea, and 121 (58.45\%) in total had menorrhagia. After a follow-up period of 23.2 months (range, 1-36 months), 119 (57.49\%) of the participants still had the LNG-IUD in place during the 3-year follow-up (Figure 2).

The baseline clinical characteristics of the 207 patients with different subtypes before treatment are presented in the Table 1. The age range of participants in subtype I was significantly older than that of subtype II and IV patients ( $\mathrm{P}=0.004$ and $\mathrm{P}=0.009$, respectively). In terms of parity, the subtype I women had more pregnancies compared to the subtype II women $(\mathrm{P}=0.002)$. The women with subtype II adenomyosis had fewer curettage procedures and a lower amount of menstrual blood loss than women in subtypes I and IV ( $\mathrm{P}=0.000$ and $\mathrm{P}=0.001$, respectively). There were no significant differences among the three groups in NRS score, body mass index, and uterine volume $(\mathrm{P}>0.05)$.

Patients from all subtypes had co-existing endometriosis and uterine fibroids, and patients in subtype II had a higher rate of pelvic endometriosis $(\mathrm{n}=56,76.71 \%)$ than the other two subtypes. The co-existing rate in the other two groups was $11.43 \%$ and $15.63 \%$ respectively. The incidence of excessive menstrual blood loss in subtypes I and IV was higher than that of subtype II $(\mathrm{P}=0.006$ and $\mathrm{P}=0.001$, respectively). Fisher's exact test showed a statistical difference in the incidence of dysmenorrhea among the three subtypes $(\mathrm{P}=0.048)$.

\section{Efficacy of the three subtypes after LNG-IUD treatment}

A total of 96 (46.38\%) patients underwent GnRHa treatment before or after LNG-IUD insertion. The injection times ranged from 1 to 6 . We also assessed patients' average NRS and PBAC scores 3-6 months after the last injection of GnRHa until LNG-IUD removal or expulsion to exclude the effect of GnRH. Table 2 shows the efficacy of LNG-IUD treatment on dysmenorrhea and menorrhagia in the three subtype groups. The NRS and PBAC scores markedly decreased after insertion of the LNG-IUD compared to baseline in the three subtypes ( $\mathrm{P}<0.001$ for all comparisons). For the 105 participants with dysmenorrhea before treatment who completed the 3-year LNG-IUD treatment, a statistically significant decrease in the NRS score was observed. Menorrhagia improved in all patients who completed the 3-year LNG-IUD treatment, and none of them had a PBAC score $>100$. 


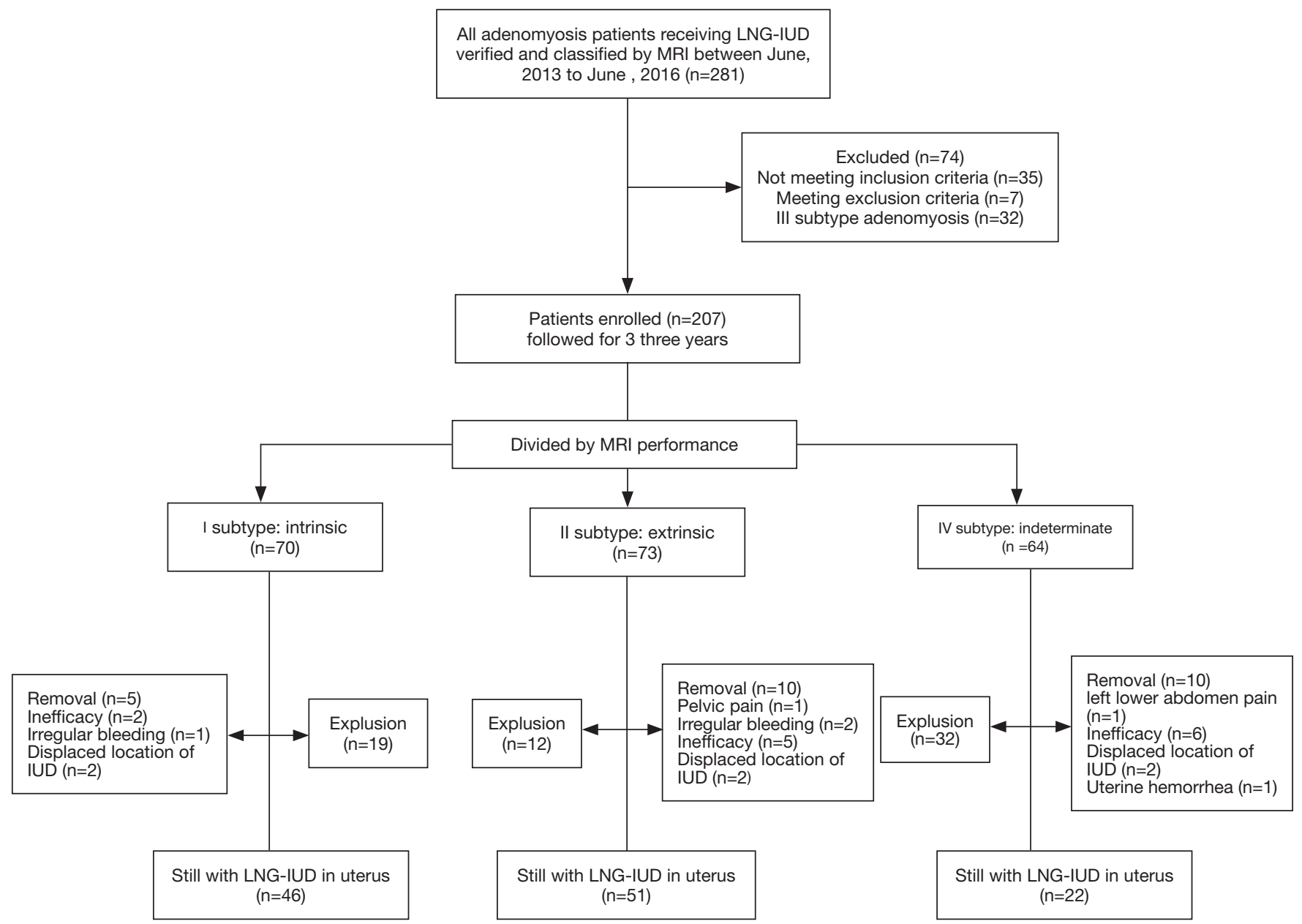

Figure 2 Flow chart of the study protocol. LNG-IUD, levonorgestrel-releasing intrauterine device; MRI, magnetic resonance imaging.

Patients who expelled the device unintentionally within 3 months after insertion were excluded in the efficiency analysis. As a result, 61, 65 and 52 patients were included in the subtype I, II, and IV groups, respectively. In terms of inefficiency, 16 (30.77\%) patients in subtype IV were found to be ineffective to such treatment, which was significantly higher compared to the other two subtypes $(\mathrm{P}=0.002$ and $\mathrm{P}=0.047$, respectively). For the recurrence of symptoms, six $(11.54 \%)$ women with subtype IV adenomyosis were found to have severe dysmenorrhea and/or heavy menstrual bleeding once again, which had ceased for a period time; the observed recurrence rate lacked statistical significance among the three subtype groups (Table 2).

\section{Analysis of device expulsion in the three subtypes}

The retention status of the LNG IUD during the follow-up period is also shown in Figure 2. The cumulative retention rates of subtype groups I, II, and IV were 46 of 70 (65.71\%), 51 of $73(69.86 \%)$, and 22 of $64(34.38 \%)$ at 3 years, respectively.

In total, 63 patients had spontaneous device expulsion within 3 years, and 57 had expulsion within 1 year in the three subtype groups. The median time of expulsion was 6.8 months (range, 1-26 months). The expulsion rates of subtypes I, II, and IV were 27.14\% (19/70), 16.44\% (12/73), and $50 \%(32 / 64)$, respectively. The subtype IV group displayed a greater expulsion rate, which was statistically significant $(\mathrm{P}<0.01$ for both comparisons), but there was no significant difference between subtypes I and II. Of the 37 participants who requested the removal of the LNGIUD, 25 expressed dissatisfaction with the treatment effect (67.57\%), four had abnormal bleeding (10.81\%), six had displaced IUD location (16.22\%), and two had related 
Table 1 The demographics and clinical characteristics of included patients

\begin{tabular}{|c|c|c|c|}
\hline Characteristics & \multicolumn{3}{|c|}{ Subtype } \\
\hline Age (years) ${ }^{\dagger}$ & $42.09 \pm 4.47^{*}$ & $39.67 \pm 4.95$ & $39.84 \pm 5.32$ \\
\hline Parity & $1.26 \pm 0.55^{\star}$ & $1.03 \pm 0.45$ & $1.13 \pm 0.55$ \\
\hline Termination of pregnancy: curettage & $2.01 \pm 1.14$ & $1.13 \pm 1.05^{\star}$ & $1.95 \pm 1.26$ \\
\hline PBAC score & $210.16 \pm 140.74$ & $123.48 \pm 99.72^{*}$ & $204.64 \pm 148.13$ \\
\hline Body mass index (BMI) & $22.58 \pm 3.35$ & $22.36 \pm 2.99$ & $23.82 \pm 12.66$ \\
\hline Uterine volume $\left(\mathrm{cm}^{3}\right)$ & $143.12 \pm 78.58$ & $141.88 \pm 75.44$ & $172.94 \pm 55.29$ \\
\hline Endometriosis, $\mathrm{n} / \mathrm{n}(\%)^{\ddagger}$ & $11.43 \%(8 / 70)$ & $76.71 \%(56 / 73)^{\star}$ & $15.63 \%(10 / 64)$ \\
\hline Menorrhagia, n/n (\%) & $70.00 \%(49 / 70)$ & $41.10 \%(30 / 73)^{\star}$ & $65.63 \%(42 / 64)$ \\
\hline
\end{tabular}

${ }^{\dagger}$, Kruskal-Wallis followed by post hoc Dunn's test or ANOVA test; ${ }^{\ddagger}, \chi^{2}$-test or Fisher's exact test. Variables presented as mean \pm standard deviation and number (percentage). ${ }^{*}, \mathrm{P}<0.05$. NRS, numeric rating scale; PBAC, pictorial blood loss assessment chart.

Table 2 Efficacy of LNG-IUD treatment on dysmenorrhea and menorrhagia

\begin{tabular}{|c|c|c|c|}
\hline Variables & \multicolumn{3}{|c|}{ Subtype } \\
\hline NRS score (before treatment) & $5.84 \pm 3.17$ & $6.82 \pm 2.64$ & $6.56 \pm 1.87$ \\
\hline NRS score (after treatment) ${ }^{\dagger}$ & $1.85 \pm 2.06^{\star \star \star}$ & $2.75 \pm 2.99^{\star \star \star}$ & $3.25 \pm 2.52^{\star \star \star}$ \\
\hline PBAC score (before treatment) & $207.38 \pm 145.70$ & $114.14 \pm 92.08$ & $183.35 \pm 134.56$ \\
\hline Invalid, $\mathrm{n} / \mathrm{n}(\%)^{\ddagger}$ & $5 / 61(8.20 \%)$ & $10 / 65(15.38 \%)$ & $16 / 52(30.77 \%)^{\star \star}$ \\
\hline Recurrence, $\mathrm{n} / \mathrm{n}(\%)$ & $2 / 61(3.28 \%)$ & $2 / 65(3.08 \%)$ & $6 / 52(11.54 \%)$ \\
\hline
\end{tabular}

${ }^{\dagger}$, Mann-Whitney $U$ tests compared to the baseline variables; ${ }^{\ddagger}, \chi^{2}$-test or Fisher's exact test. Data were presented as mean \pm standard deviation and number (percentage). ${ }^{* \star}, \mathrm{P}<0.01 ;{ }^{* \star}, \mathrm{P}<0.001$. LNG-IUD, levonorgestrel-releasing intrauterine device; NRS, numeric rating scale; PBAC, pictorial blood loss assessment chart.

pelvic or abdominal pain (5.41\%).

The results of the univariate and multivariate Cox regression analyses revealed that the uterine volume before the LNG-IUD insertion was the independent factor associated with spontaneous expulsion of the device in the subtype $\mathrm{I}$ adenomyosis patients ( $\mathrm{P}=0.029$, HR: 3.37 , $95 \%$ CI: 1.09-6.88). In the subtype II group, device expulsion had no statistically significant relationship with any factors, including treatment with GnRHa. After adjustment of other related factors, the amount of menstrual blood loss after Mirena became an independent factor for maintaining the
LNG-IUD in subtype IV patients ( $\mathrm{P}=0.045$, HR: $1.02,95 \%$ CI: 1.01-1.21) (Tables 3 and 4).

\section{Menstrual bleeding pattern changes and evaluation of side-effects}

Abnormal uterine bleeding was the most frequent complaint regarding menstrual pattern; however, this symptom generally improved 1 year after the device was placed in the uterine cavity. The menstrual pattern complaints among adenomyosis patients subtype I was prolonged bleeding 
Table 3 Univariate analysis of risk factors for LNG-IUD expulsion in the treatment of adenomyosis

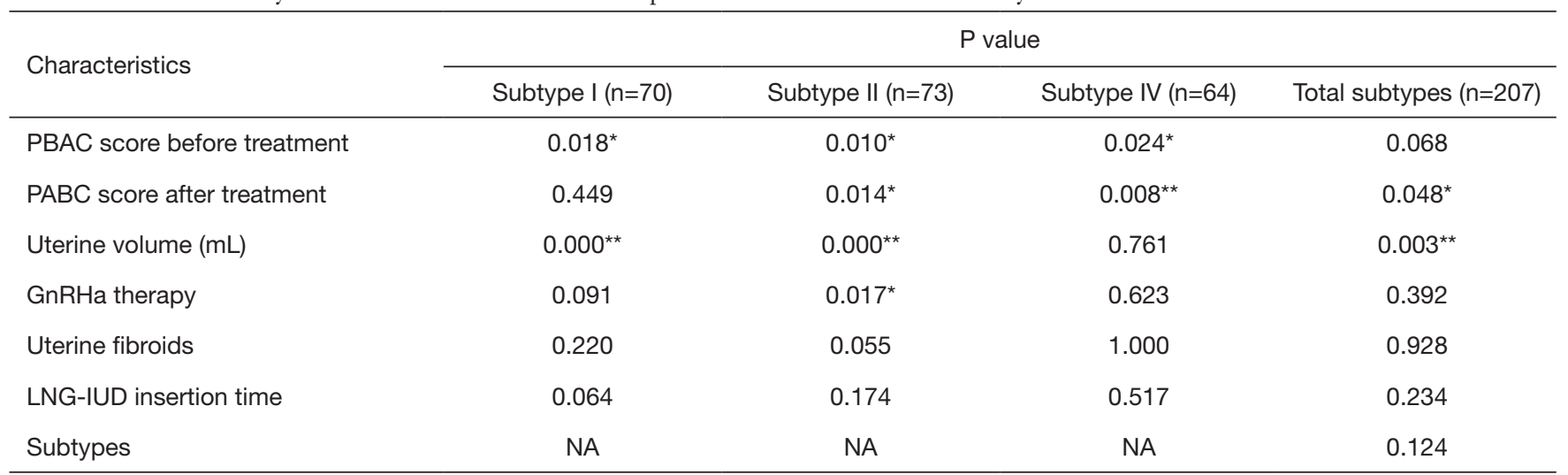

Univariate analysis. *, $\mathrm{P}<0.05$; ${ }^{*}, \mathrm{P}<0.01$. PBAC, pictorial blood loss assessment chart; GnRHa, gonadotropin-releasing hormone agonist; LNG-IUD, levonorgestrel-releasing intrauterine device; NA, not applicable.

Table 4 Multifactor Cox regression analyses of risk factors for LNG-IUD expulsion in the treatment of adenomyosis

\begin{tabular}{|c|c|c|c|c|c|c|c|c|}
\hline Characteristics & \multicolumn{2}{|c|}{ Subtype I } & \multicolumn{2}{|c|}{ Subtype II } & \multicolumn{2}{|c|}{ Subtype IV } & \multicolumn{2}{|c|}{ Total subtypes } \\
\hline $\begin{array}{l}\text { PBAC score before } \\
\text { treatment }\end{array}$ & 0.676 & NA & 0.566 & NA & 0.188 & NA & 0.971 & NA \\
\hline $\begin{array}{l}\text { PABC score after } \\
\text { treatment }\end{array}$ & NA & NA & 0.925 & NA & $0.045^{\star}$ & $1.02(1.01-1.21)$ & 0.080 & NA \\
\hline GnRHa therapy & NA & NA & 0.063 & NA & 0.161 & NA & 0.480 & NA \\
\hline Uterine fibroids & NA & NA & 0.676 & NA & NA & NA & NA & NA \\
\hline $\begin{array}{l}\text { LNG-IUD insertion } \\
\text { time }\end{array}$ & 0.241 & NA & NA & NA & NA & NA & NA & NA \\
\hline
\end{tabular}

${ }^{\dagger}$, multi-factors Cox regression analysis. *, P<0.05. PBAC, pictorial blood loss assessment chart; GnRHa, gonadotropin-releasing hormone agonist; LNG-IUD, levonorgestrel-releasing intrauterine device; NA, not applicable; HR, hazard ratio; Cl, confidence intervals.

(7/46, 15.22\%), amenorrhea (9/46, 19.57\%), and irregular bleeding (4/46, 8.70\%). One patient gained $>5 \mathrm{~kg}$ during the treatment, and the incidence of abnormal location device was $4.35 \%(2 / 46)$. Menstrual changes mainly accounted for amenorrhea in $11.76 \%(6 / 51)$ of cases and prolonged bleeding in $13.73 \%$ (7/51) of cases in the subtype II group. Recurrent vaginitis occurred in one case in the subtype II group. The menstrual patterns of the patients with subtype IV adenomyosis included prolonged menstrual and irregular bleeding, and severe acne occurred in one patient.

\section{Discussion}

MRI is considered highly accurate in the diagnosis of adenomyosis, as well as in differentiation from other intrauterine gynecological diseases, such as leiomyoma (9). In contrast to TVS, MRI has the advantages of excellent soft tissue differentiation and less operatordependent interpretation. Moreover, several prospective research studies have found a higher diagnostic accuracy of sensitivity (77-93\%) and specificity (86-93\%) in the diagnosis of adenomyosis by MRI compared to other techniques $(3,15)$. An increasing number of studies have focused on the classification of adenomyosis, depending on MRI characteristics. In 2012, Kishi et al. stated that uterine adenomyosis could be differentiated into four subtypes based on the relationship between occurring sites and other structural components: intrinsic, extrinsic, intramural, and indeterminate (4). In the present study, we excluded the subtype III of adenomyosis due to the feasibility and efficacy 
of adenomyomectomy, which is similar to uterine fibroids removal surgery.

It has been speculated that each disease subtype has a different origin and pathology, and eventually displays specific clinical and biochemical features (16). The classically accepted hypothesis is that tissues injure and repair mechanisms that causes direct invasion and migration of the endometrial basalis layer into the myometrium in intrinsic adenomyosis (17). The increased local production of estradiol in tissue auto-traumatization and hyperperistaltic uterine activity mediated by oxytocin leads to increased mechanical stresses that injure the endometrium within the junctional zone, consequently resulting in the formation of adenomyotic lesions (18). In our study, curettage procedures and parity were found to be more common in subtype I patients, which is in agreement with previously published reports $(4,16)$. Barrier damage by repeated surgical interventions and trophoblastic invasion during pregnancy allows the endometrium to directly invade the myometrium.

In contrast, the epithelial-mesenchymal transition has been reported to be implicated in the pathogenesis of extrinsic uterine adenomyosis, characterized by the activation of transforming growth factor (TGF)- $\beta 1$ through the Smad2/3dependent signaling pathway (19). Subtype II adenomyosis is believed to have both a direct and indirect influence on endometriosis or a subtype of endometriosis. Donnez et al. found that external adenomyosis patients had a high rate (97\%) of deep infiltrating endometriosis (20). Khan et al. reported that extrinsic adenomyosis has a close biological and histological correlation with co-existent deep infiltrating endometriosis (21). It is highly suspect of a shared drug treatment protocol for long-term administration in two diseases. However, $76.71 \%$ of the subtype II adenomyosis cases had concurrence pelvic endometriosis in our study, which was less than that found by other researchers. We speculated that there could be another pathogenic process in subtype II adenomyosis.

The subtype IV adenomyosis group, which included patients who did not meet any of the other three classification criteria, was a heterogeneous mixture of subtypes I-III. Generally, subtype IV has extensive and diverse lesions, which could support the high rates of dysmenorrhea and menorrhagia. It had been speculated that the subtype IV adenomyosis is an advanced stage of the disease. In our study, the mean uterine volume of this subtype was more significant than that of subtypes I and II adenomyosis; however, there was no statistically significant difference.
It is well known that the LNG-IUD is used to relieve dysmenorrhea and the heavy menstrual bleeding associated with adenomyosis $(8,22)$. Our study extended the research by exploring the relationship between the classification of adenomyosis by MRI and the effect of the LNG-IUD. The NRS and PBAC scores of the patients in the three subtype groups were all significantly reduced after LNGIUD insertion. The overall 3 -year efficacy rate was $88.52 \%$, $81.54 \%$ and $57.69 \%$ for subtypes I, II, and IV, respectively; this finding is consistent with published studies concerning relief from pain and the amount of menstrual blood loss. However, subtype IV patients had a high incidence of failure and recurrence in this treatment. The current findings of persistent low progesterone receptor expression and the strong distribution of fibrosis around adenomyotic foci in the myometrium may explain the poor response to levonorgestrel medication in the subtype IV adenomyosis group (23). Fibrosis is a time-dependent process to extend to the normal myometrium around; therefore, we should reconsider the origin and pathogenesis of this phenotype. We suggest that it is necessary to choose a more appropriate treatment approach to cure this subtype disease. The combination of the LNGIUD with HIFU or conservative surgery may demonstrate a superior clinical effect for this subtype (24).

In the present study, the cumulative LNG-IUD retention rate was $57.49 \%$ for the 207 patients in the three groups, which was similar to the rate reported by $\mathrm{Li}$ et al. (22). However, the spontaneous expulsion of LNGIUD increased dissatisfaction among patients. The overall discharge rate was $30.43 \%$ in the groups. Also, the majority of expulsions occurred within 6 months after device insertion and reached a steady status after 1 year. It should be noted that previous expulsions were associated with a significantly higher risk for a re-expulsion when inserting the IUD (25). As a result, it is vital to improving the initial LNG-IUD treatment to avoid re-expulsion.

In our study, $50 \%$ of the 62 women in subtype IV group complained of spontaneous expulsion of the device within 3 years after insertion. Lee $e t$ al. reported that the rate of LNG-IUD expulsion was related to uterine volume. The failure rate was significantly increased when the uterine volume was $>150 \mathrm{~mL}$ (12). Several published studies found that the application of GnRHa could markedly improve the device expulsion rate $(22,26)$. In the present study, there was a relationship between uterine volume and LNG-IUD expulsion in subtype I women, which was statistically significant. It was highly speculated that the greater lesion size, the greater deformation in uterine cavity 
morphology in intrinsic adenomyosis. In contrast, blood loss amount after LNG-IUD insertion was associated with spontaneous discharge in subtype IV adenomyosis. In our analysis, treatment with GnRHa for variable periods of 1-6 months failed to demonstrate a positive effect on expulsion improvement. We still emphasize the value of GnRHa therapy in reducing the size of the lesions and the amount of blood loss before or after the IUD insertion.

\section{Limitations}

The present study had some limitations. First, it was a retrospective, and single-center study with inevitable recall bias, so unknown confounding factors could affect the outcome. Further, the study population size was small due to many factors, such as the relative rarity of MRI before LNG-IUD insertion. Third, concerning the device expulsion analysis, more possible elements should be taken into account, such as dysmenorrhea. A large-scale prospective and randomized study with a longer follow-up time is required to validate our results.

\section{Conclusions}

Adenomyosis can be identified by MRI and has different clinical and biologic characteristics: intrinsic, extrinsic, intramural and indeterminate. The LNG-IUD is a practical approach to treat subtypes I and II adenomyosis. The spontaneous expulsion of the LNG-IUD was related to uterine volume and the amount of menstrual blood loss in subtypes I and IV adenomyosis. Due to the high incidence of treatment failure and expulsion, further study is warranted to explore a more suitable protocol to treat subtype IV adenomyosis for long-term administration.

\section{Acknowledgments}

We deeply appreciate the patients who gave great help to the study.

Funding: The work was supported by National Key R\&D Program of China (grant number: 2017YFC1001202) and National Natural Science Foundation of China (grant numbers: 81671429 and 81974225 ).

\section{Footnote}

Reporting Checklist: The authors have completed the STROBE reporting checklist. Available at: http://dx.doi. org/10.21037/atm-20-3420

Data Sharing Statement: Available at http://dx.doi. org/10.21037/atm-20-3420

Peer Review File: Available at http://dx.doi.org/10.21037/ atm-20-3420

Conflicts of Interest: All authors have completed the ICMJE uniform disclosure form(available at: http://dx.doi. org/10.21037/atm-20-3420). XZ reports that he serves as an unpaid editorial board member of Annals of Translational Medicine from Mar/2020 to now. The other authors have no conflicts of interest to declare.

Ethical Statement: The authors are accountable for all aspects of the work in ensuring that questions related to the accuracy or integrity of any part of the work are appropriately investigated and resolved. The Ethics Committee of Women' s Hospital, Zhejiang University School (No. IRB-20190089012) approved the study in accordance with the ethical standards in the Declaration of Helsinki (as revised in 2013) and waived the informed consent.

Open Access Statement: This is an Open Access article distributed in accordance with the Creative Commons Attribution-NonCommercial-NoDerivs 4.0 International License (CC BY-NC-ND 4.0), which permits the noncommercial replication and distribution of the article with the strict proviso that no changes or edits are made and the original work is properly cited (including links to both the formal publication through the relevant DOI and the license). See: https://creativecommons.org/licenses/by-nc-nd/4.0/.

\section{References}

1. Exacoustos C, Zupi E. A new era in diagnosing adenomyosis is coming. Fertil Steril 2018;110:858.

2. Van den Bosch T, Dueholm M, Leone FPG, et al. Terms, definitions and measurements to describe sonographic features of myometrium and uterine masses: a consensus opinion from the Morphological Uterus Sonographic Assessment (MUSA) group. Ultrasound Obstet Gynecol 2015;46:284-98.

3. Bazot $M$, Darai E. Role of transvaginal sonography and magnetic resonance imaging in the diagnosis of uterine adenomyosis. Fertil Steril 2018;109:389-97.

4. Kishi Y, Suginami H, Kuramori R, et al. Four subtypes 
of adenomyosis assessed by magnetic resonance imaging and their specification. Am J Obstet Gynecol 2012;207:114.e1-7.

5. Kobayashi H, Matsubara S. A Classification Proposal for Adenomyosis Based on Magnetic Resonance Imaging. Gynecol Obstet Invest 2020;85:118-26.

6. Vannuccini S, Luisi S, Tosti C, et al. Role of medical therapy in the management of uterine adenomyosis. Fertil Steril 2018;109:398-405.

7. Neriishi K, Hirata T, Fukuda S, et al. Long-term dienogest administration in patients with symptomatic adenomyosis. J Obstet Gynaecol Res 2018;44:1439-44.

8. Sheng J, Zhang WY, Zhang JP, et al. The LNG-IUS study on adenomyosis: a 3-year follow-up study on the efficacy and side effects of the use of levonorgestrel intrauterine system for the treatment of dysmenorrhea associated with adenomyosis. Contraception 2009;79:189-93.

9. Tan J, Yong P, Bedaiwy MA. A critical review of recent advances in the diagnosis, classification, and management of uterine adenomyosis. Curr Opin Obstet Gynecol 2019;31:212-21.

10. Breivik H, Borchgrevink PC, Allen SM, et al. Assessment of pain. Br J Anaesth 2008;101:17-24.

11. Higham JM, Obrien PMS, Shaw RW. Assessment of Menstrual Blood-Loss Using a Pictorial Chart. Br J Obstet Gynaecol 1990;97:734-9.

12. Lee KH, Kim JK, Lee MA, et al. Relationship between uterine volume and discontinuation of treatment with levonorgestrel-releasing intrauterine devices in patients with adenomyosis. Arch Gynecol Obstet 2016;294:561-6.

13. Wu J, Huang Y, Chen L, et al. Treatment of Adenomyosis with Subcutaneous Etonogestrel Implants: A Clinical Observational Study in 17 Patients. Med Sci Monit 2018;24:6085-92.

14. Zhu L, Chen S, Che X, et al. Comparisons of the efficacy and recurrence of adenomyomectomy for severe uterine diffuse adenomyosis via laparotomy versus laparoscopy: a long-term result in a single institution. J Pain Res 2019;12:1917-24.

15. Champaneria R, Abedin P, Daniels J, et al. Ultrasound scan and magnetic resonance imaging for the diagnosis of adenomyosis: systematic review comparing test accuracy. Acta Obstetricia Et Gynecologica Scandinavica 2010;89:1374-84.

16. Kishi Y, Shimada K, Fujii T, et al. Phenotypic characterization of adenomyosis occurring at the inner and outer myometrium. PLoS One 2017;12:e0189522.
17. Leyendecker G, Wildt L. A new concept of endometriosis and adenomyosis: tissue injury and repair (TIAR). Horm Mol Biol Clin Investig 2011;5:125-42.

18. Ibrahim MG, Sillem M, Plendl J, et al. Myofibroblasts Are Evidence of Chronic Tissue Microtrauma at the Endometrial-Myometrial Junctional Zone in Uteri With Adenomyosis. Reprod Sci 2017;24:1410-8.

19. Shen M, Liu X, Zhang H, et al. Transforming growth factor beta1 signaling coincides with epithelialmesenchymal transition and fibroblast-to-myofibroblast transdifferentiation in the development of adenomyosis in mice. Hum Reprod 2016;31:355-69.

20. Donnez J, Dolmans MM, Fellah L. What if deep endometriotic nodules and uterine adenomyosis were actually two forms of the same disease? Fertility and Sterility 2019;111:454-6.

21. Khan KN, Fujishita A, Koshiba A, et al. Biological differences between intrinsic and extrinsic adenomyosis with coexisting deep infiltrating endometriosis. Reprod Biomed Online 2019;39:343-53.

22. Li L, Leng J, Jia S, et al. Treatment of symptomatic adenomyosis with the levonorgestrel-releasing intrauterine system. Int J Gynaecol Obstet 2019;146:357-63.

23. Khan KN, Fujishita A, Koshiba A, et al. Biological differences between focal and diffuse adenomyosis and response to hormonal treatment. Reprod Biomed Online 2019;38:634-46.

24. Haiyan S, Lin W, Shuhua H, et al. High-intensity focused ultrasound (HIFU) combined with gonadotropin-releasing hormone analogs (GnRHa) and levonorgestrel-releasing intrauterine system (LNG-IUS) for adenomyosis: a case series with long-term follow up. Int J Hyperthermia 2019;36:1179-85.

25. Merki-Feld GS, Schwarz D, Imthurn B, et al. Partial and complete expulsion of the Multiload 375 IUD and the levonorgestrel-releasing IUD after correct insertion. Eur J Obstet Gynecol Reprod Biol 2008;137:92-6.

26. Park DS, Kim ML, Song T, et al. Clinical experiences of the levonorgestrel-releasing intrauterine system in patients with large symptomatic adenomyosis. Taiwan J Obstet Gynecol 2015;54:412-5.

Cite this article as: Chen S, Wang J, Sun W, Zhu L, He J, Zhang X. Efficacy of the levonorgestrel-releasing intrauterine device is associated with different subtypes of adenomyosis: a retrospective study. Ann Transl Med 2020;8(21):1356. doi: 10.21037/atm-20-3420 